\title{
Investigation of MIMO Channel Characteristics in a Two-Section Tunnel at $1.4725 \mathrm{GHz}$
}

\author{
Rongchen Sun, ${ }^{1}$ David W. Matolak, ${ }^{2}$ Cheng Tao, ${ }^{1}$ Liu Liu, ${ }^{1}$ Zhenhui Tan, ${ }^{1}$ and Tao Zhou ${ }^{1}$ \\ ${ }^{1}$ Institute of Broadband Wireless Mobile Communications, Beijing Jiaotong University, Beijing 100044, China \\ ${ }^{2}$ Department of Electrical Engineering, University of South Carolina, Columbia, SC 29208, USA
}

Correspondence should be addressed to Tao Zhou; taozhou@bjtu.edu.cn

Received 24 February 2017; Revised 24 May 2017; Accepted 6 June 2017; Published 10 July 2017

Academic Editor: Sotirios K. Goudos

Copyright (C) 2017 Rongchen Sun et al. This is an open access article distributed under the Creative Commons Attribution License, which permits unrestricted use, distribution, and reproduction in any medium, provided the original work is properly cited.

\begin{abstract}
This paper presents results from a wide band single-input-single-output (SISO) and $16 \times 16$ virtual multiple-input-multiple-output (MIMO) measurement campaign at a center frequency of $1.4725 \mathrm{GHz}$ in a 100 -meter long tunnel laboratory which is terminated by a vertical wall with a metallic door. The path loss, root-mean-square delay spread (RMS-DS) characteristics, and power delay profiles (PDPs) are described. In addition, we provide results for the MIMO channel amplitude matrix, which offers a new perspective in understanding MIMO characteristics in tunnel scenarios. Our measurement results are analyzed and compared to ray tracing simulations. The relationships among the angle spread, channel matrix singular values, and MIMO capacity at various link distances are illustrated, and these provide insights into MIMO system deployment.
\end{abstract}

\section{Introduction}

To ease the growing population pressure on urban traffic, major cities in China have accelerated subway construction. It is expected that by 2020 more than 45 cities in China will have subways, and all new subways under construction must have a large rush hour capacity. Increasing train running speed and reducing the interval between adjacent trains enable a passenger capacity increase, but at the same time this introduces some security risks such as those due to train malfunction or other disasters (e.g., fires). Thus, these trains require various levels of monitoring to ensure safe and efficient operation. For example, video information should be obtained in real time at various locations.

A wide band and MIMO wireless communication system can satisfy the increasing data transmission requirements for both train control systems and passenger communications. Before any MIMO system is deployed underground, the MIMO channel should be accurately characterized. Much work in the literature has discussed MIMO link performance by measurement and various theoretical methods.

Extensive work has been done on propagation and MIMO channel capacity based on measurements in subway tunnels. Using electromagnetic field theory, for example, [1-6], researchers have confirmed that strong wave guiding effects make the tunnel channel characteristics different from those in other scenarios such as indoor scenario. The authors of [7, 8] provided some wide band measurement results, including time delay dispersion and Ricean $\mathrm{K}$ factors. In $[1,2]$ the authors noted that antenna alignment and the shape of the tunnel cross section have a great influence on the correlations between antennas, the channel matrix singular values, and MIMO capacity. However, to fully explore the advantages of using a large number of antennas in the tunnel, more detailed information regarding medium or large scale MIMO system performance is required. Additionally, dense measurements in the spatial domain also provide information that may be used to improve MIMO capacity when there are limits to antenna alignment precision or antenna selection algorithms [9].

The authors of $[10,11]$ regarded the tunnel as a nonideal waveguide, but this makes it difficult to consider various factors such as the effects of cross section shape, curves, wall materials, and metal reflecting objects in the tunnel. Therefore, some authors employ ray tracing, for example, $[12,13]$. Nonetheless, more work should be done to verify the simulation accuracy through comparison with measurements. This paper focuses on such a comparison of 

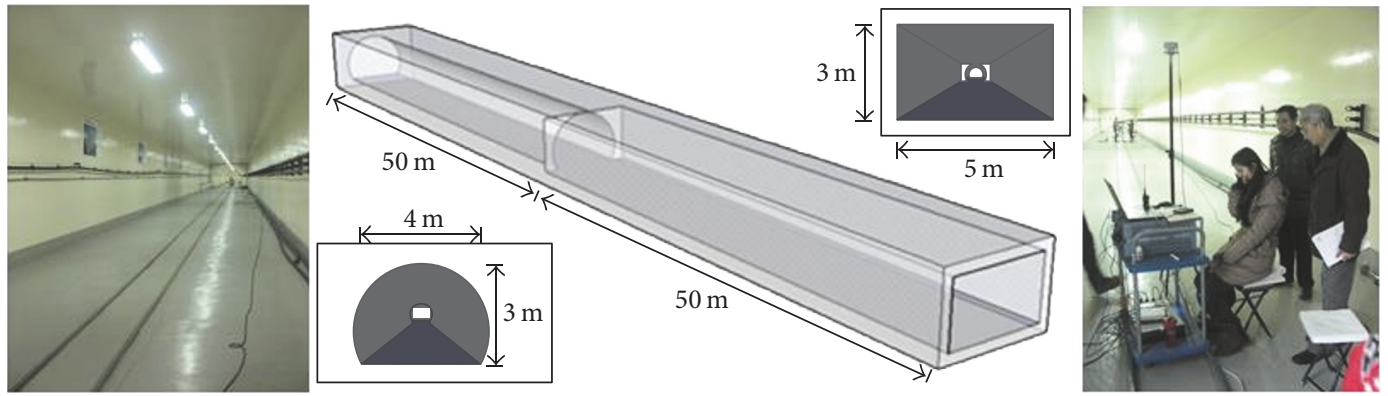

Figure 1: Tunnel laboratory in Zhongtian Technology Group.

wide band MIMO channel characteristics with ray tracing results.

The organization of the paper is as follows. In Section 2, the measurement campaign is summarized. In Section 3, the measurement results, including path loss, time delay dispersion, and the array amplitude matrix, are analyzed and compared with ray tracing results. The relationships among the angle spread, singular values, and channel capacity are also illustrated. Section 4 presents the conclusion.

\section{Description of the Measurement Campaign}

2.1. Measurement Environment. Our measurement campaign was conducted in the Tunnel Laboratory in Zhongtian Technology Group Company, which is located in Nantong City, Jiangsu Province. The laboratory was built to test the performance of the company's leaky cables. The tunnel is made of reinforced concrete and consists of two parts of different cross-sectional shape, rectangular and circular; see Figure 1. The length of each part is $50 \mathrm{~m}$. The interior width and height of the rectangular part are $5 \mathrm{~m}$ and $3 \mathrm{~m}$, respectively. The interior radius of the circular part is $2 \mathrm{~m}$ and the height is $3 \mathrm{~m}$; the circular section is truncated at its base. The receiver is fixed at the end of the rectangular part and the transmitter was moved to several locations in the tunnel.

2.2. Measurement Equipment. The measurement system is shown in Figure 2. The transmitter (Tx) is a Rohde \& Schwarz commercial signal generator SMBV100A. The receiver was made by our research group at Beijing Jiaotong University using several components. This receiver $(\mathrm{Rx})$ equipment can acquire and store the IF signal after downconversion by the RF module. A GPS synchronization signal is transmitted by an optical fiber to both Tx and Rx. The antenna is a biconical antenna. This measurement system is mainly used in virtual MIMO measurements. Table 1 lists the primary measurement system parameters.

2.3. Probe Signal and Data Processing. Zadoff-Chu (ZC) sequences are often used as synchronization signals due to their good orthogonality and low peak-to-average power ratio properties. The complex value of each sequence is given by [14]

$$
a_{q}=\exp \left[-j 2 \pi q \frac{n(n+1) / 2+l n}{N_{\mathrm{ZC}}}\right],
$$

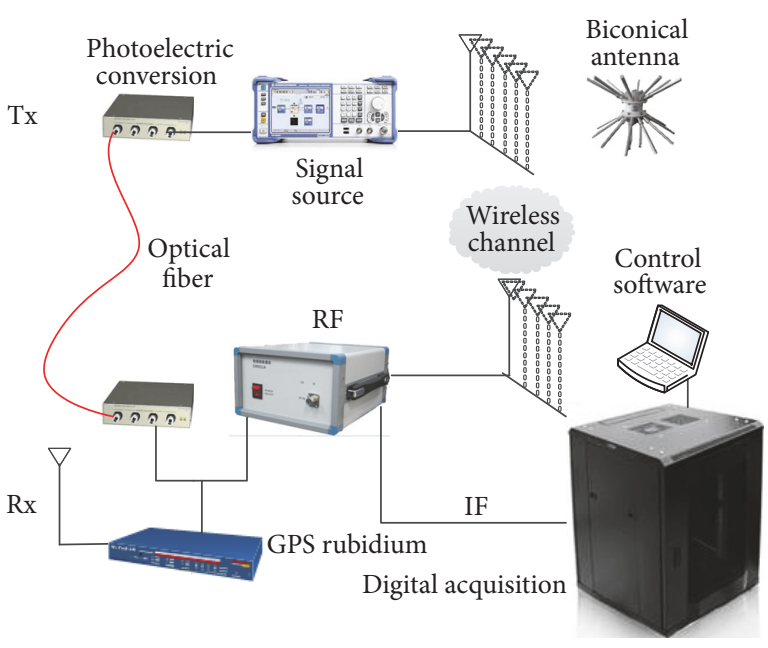

FIgURE 2: Measurement system.

where odd number $N_{\mathrm{ZC}}$ is the sequence length, and $q \epsilon$ $\left\{1, \ldots, N_{\mathrm{ZC}}-1\right\}$ is the sequence root index. Parameter $n=$ $0,1, \ldots, N_{\mathrm{ZC}}-1$ is an index within the sequence, and different sequences can be created by changing $l \in Z$. In this paper, a $\mathrm{ZC}$ sequence generated by $q=1, l=33$, and $N_{\mathrm{Zc}}=2047$ is used as the probe signal.

The channel impulse response (CIR) $h\left[\tau_{m}\right]$ can be obtained by sliding correlation of the received signal and local $\mathrm{ZC}$ sequence as

$$
h\left[\tau_{m}\right]=\frac{1}{N_{\mathrm{sc}}} \sum_{k=0}^{N_{\mathrm{sc}}-1} r\left[t_{k}\right] \cdot c\left[t_{k}-\tau_{m}\right] d t,
$$

where $r\left[t_{k}\right]$ is received signal at time $t_{k}, \tau$ is the multipath delay, $k$ and $m$ are time and delay indices, and $c\left(t_{k}\right)$ is the locally generated $\mathrm{ZC}$ sequence.

As noted, the length of the $\mathrm{ZC}$ sequence used here is 2047. Hence the maximum delay that can be measured is $(1 / 91 \mathrm{MHz}) \times 2047=2.25 \times 10^{-5} \mathrm{~s}$. This value is much larger than the delay where any echoes can incur within the tunnel. So the receiver noise threshold can be estimated by the last (long-delay) part of the PDP where no echoes can exist. Since the noise threshold varies with $\mathrm{Tx}$ - $\mathrm{Rx}$ distance, a fixed threshold $-40 \mathrm{~dB}$ relative to the power delay profile (PDP) peak 
TABLE 1: Measurement parameters.

\begin{tabular}{lc}
\hline Center frequency & $1.4725 \mathrm{GHz}$ \\
Signal bandwidth & $91 \mathrm{MHz}$ \\
Antenna type & Biconical \\
Antenna pattern & Omnidirectional (azimuth) \\
& SISO \\
Antenna deployment & Virtual MIMO $16 \times 16$, spacing \\
& $\lambda / 2$ \\
Probe signal & ZC sequence, length 2047 \\
Transmit power & $10 \mathrm{dBm}$ \\
\hline
\end{tabular}

is chosen for declaring valid multipath components. Any components greater than the noise threshold are assumed to be valid multipath components.

\section{Measurement Results}

\subsection{SISO Measurement}

3.1.1. Path Loss. To measure the path loss (PL) in the tunnel, the Tx was moved from one end to the other in $1 \mathrm{~m}$ intervals. The $\mathrm{PL}$ is given by

$$
\mathrm{PL}=P_{\mathrm{Rx}}-P_{\mathrm{Tx}}
$$

where $P_{\mathrm{Tx}}$ is transmit power and $P_{\mathrm{Rx}}$ is received power, which is given by $[15,16]$

$$
P_{\mathrm{Rx}}=\left|\sum_{m=1}^{L} h\left[\tau_{m}\right]\right|^{2},
$$

where $L$ is the number of valid multipath components (MPCs).

The path loss results obtained from the SISO measurement and those from the free space model and ray tracing simulations, all versus linear distance, are shown in Figure 4. The ray tracing tool we used here is Wireless InSite. X3D model was chosen in the simulation and diffractions are included. The accuracy in the ray tracing simulation depends on the reconstruction of the measurement environment and parameter settings established prior to simulation. Thus the ray tracing result is only approximate due to imperfect representation of the tunnel structure and electrical properties. The relative permittivity and conductivity values are 5 and $0.001 \mathrm{~S} / \mathrm{m}$, respectively. The number of reflections accounted for in the ray tracer is 8 , and no more than 300 rays are employed in the simulation.

The path loss can be divided into two sections: before and after a distance of approximately $45 \mathrm{~m}$. For the short range region, the measured path loss is up to $70 \mathrm{~dB}$. In this short range section, the measured path loss is similar to, but slightly less than, the path loss of free space. After reaching $70 \mathrm{~dB}$ at $45 \mathrm{~m}$, the measured path loss fluctuates around a value of approximately $60 \mathrm{~dB}$. The drop at $45 \mathrm{~m}$ hence appears to come from the reduction of the cross section at the middle of the tunnel (transition from rectangular to circular), where the waveguide effect evidently increases; this

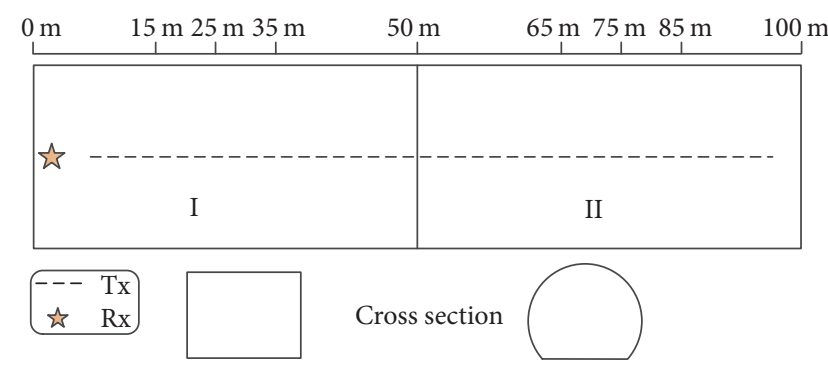

FIgURE 3: SISO path loss measurement.

waveguide effect is also expected to be stronger at longer link distances. The steady trend after $45 \mathrm{~m}$ is attributable to low order reflected multipath components (MPCs) adding to the LOS component; these MPCs are strongly affected by waveguide effect. The ray tracing results exhibit the same basic trend as the measurements but show more fluctuations in both sections. We point out that these path loss results did not average out small scale fading effects; hence the results are not used for path loss modeling but rather to observe overall trends in this two-section tunnel.

\subsubsection{RMS Delay Spread. The RMS-DS is given by [15]}

$$
\sigma_{\mathrm{rms}}=\sqrt{\overline{\overline{\tau^{2}}-(\bar{\tau})^{2}}}
$$

where $\overline{\tau^{2}}$ and $\bar{\tau}$ can be written as

$$
\begin{gathered}
\overline{\tau^{2}}=\frac{\sum_{k} P\left(\tau_{k}\right) \tau_{k}^{2}}{\sum_{k} P\left(\tau_{k}\right)}, \\
\bar{\tau}=\frac{\sum_{k} P\left(\tau_{k}\right) \tau_{k}}{\sum_{k} P\left(\tau_{k}\right)},
\end{gathered}
$$

where $P(\tau)$ is the power measured at delay $\tau$ (the PDP) and the mean excess delay $\bar{\tau}$ is the first moment of the PDP.

Figure 5 shows the RMS-DS results versus link distance from measurements and ray tracing with and without the reflection from the rear door (wall at $100 \mathrm{~m}$ in Figure 3); each point represents a RMS-DS result obtained from an individual PDP. In Figure 5(a), both curves show the same general trend of gradual rising and then falling back gradually; this agrees with intuition in such a "closed" structure. The maximum RMS-DS is approximately 150 to $160 \mathrm{~ns}$ at the middle of the tunnel and after a link distance of 60 to $70 \mathrm{~m}$, the RMS-DS rapidly declines to about 20 ns. Figure 5(b) illustrates that after removing the rear door reflection, the RMS-DS stays at a low value of approximately $20 \mathrm{~ns}$. Clearly any such long reflections as those from the rear door will have a strong influence on RMS-DS. As we can see, in both Figures 5(a) and 5(b), that there are deviations between measurement and ray tracing results, particularly at positions 10 to $20 \mathrm{~m}$, 25 to $40 \mathrm{~m}$, and 65 to $75 \mathrm{~m}$. We repeated the ray tracing simulations a number of times, adjusting the number of reflections and the tunnel wall material parameters to try to improve agreement. In the ray tracing simulation, increasing the number of reflections will increase the time delay, so 


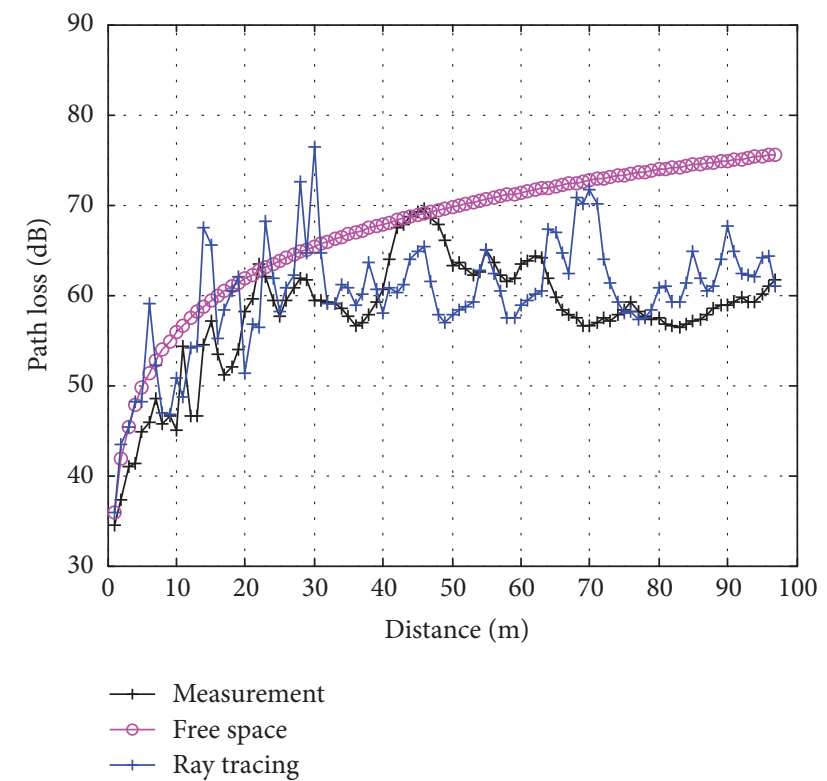

FIgURE 4: Path loss versus distance for measurement, ray tracing, and free space results.

we found that 8 reflections are appropriate for reaching a reasonable match between measurement and simulation. Also it is worth pointing out that the tunnel walls are not perfectly smooth, and since we did not have detailed information on the tunnel construction, we could not model the detailed material structure of the walls (e.g., the possible presence of reinforcing metal rods, so-called "rebar"). As seen in comparing Figure 5(a) with Figure 5(b), removing the back wall reflections does improve the agreement between measurements and simulations. The normalized RMS error $\widetilde{\varepsilon}_{\text {RMSE }}$ between measurement and ray tracing results is given by

$$
\widetilde{\varepsilon}_{\mathrm{RMSE}}=\sqrt{\frac{\left(\widetilde{\sigma}_{\mathrm{rmsM}}-\widetilde{\sigma}_{\mathrm{rmsR}}\right)^{2}}{N_{\mathrm{rms}}}},
$$

where $\widetilde{\sigma}_{\text {rmsM }}$ and $\widetilde{\sigma}_{\text {rmsR }}$ are RMS-DS values that are normalized by the mean RMS-DS in each case. The normalized RMS errors in case of Figures 5(a) and 5(b) are 0.70 and 0.42, respectively.

3.2. Virtual MIMO Measurement. As Figure 6 shows, the channel was measured at six positions along lines orthogonal to the tunnel longitudinal axis, at several link distances. At each position, 16 by $16 \mathrm{MIMO}$ are used and at link distances of $25 \mathrm{~m}$ and $75 \mathrm{~m}$, three Tx antenna heights $(2.3 \mathrm{~m}, 2.4 \mathrm{~m}$, and $2.5 \mathrm{~m}$ ) were used.

3.2.1. PDP Results. Figure 7 is a measurement and ray tracing PDP example when the Tx was placed at $25 \mathrm{~m}$. Because the tunnel is closed, the echoes from the walls of the end of the tunnel can be found in the measured CIRs. As Figure 7 shows, part $A$ is the useful signal, the echoes of part $B$ are from the junction of the two tunnels, and part $\mathrm{C}$ is the reflection from the rear-wall and metal door at the end of tunnel II. It is
TABLE 2: Number of multipath components using fixed threshold and SAGE.

\begin{tabular}{lcccccc}
\hline Position & $15 \mathrm{~m}$ & $25 \mathrm{~m}$ & $35 \mathrm{~m}$ & $65 \mathrm{~m}$ & $75 \mathrm{~m}$ & $85 \mathrm{~m}$ \\
\hline Fixed threshold & 9 & 12 & 12 & 12 & 9 & 9 \\
SAGE & 12 & 13 & 12 & 12 & 10 & 10 \\
\hline
\end{tabular}

obvious that in the ray tracing PDP, echoes in parts B and C can be distinguished from those in part A easily, according to the time delay. However, as link distance increases, the MPCs of parts B and C come gradually closer to those of part A, which makes it difficult to identify the valid MPCs of part A from the PDP. Thus, in cases like the PDP at $25 \mathrm{~m}$ in Figure 7, MPCs of parts B and C can be removed completely, but in cases like the PDP at $85 \mathrm{~m}$ (shown in Figure 13), we can only remove the signal under the noise floor, in which case some reflections from the rear door cannot be removed completely, and this will have some influence on the subsequent parameter analysis. This discussion also pertains to the RMS-DS results in Figure 5.

The measured PDPs for link distances from $15 \mathrm{~m}$ to $85 \mathrm{~m}$ appear in Figures 8-13. All the PDPs in these figures pertain to the case when both the Tx and Rx antennas are located near the tunnel center. Here number 8 transmit antenna to number 8 receiving antenna PDPs are shown.

In Figures 8-13, it can be observed that most of the useful signal has delay less than $200 \mathrm{~ns}$, and as the distance increases, the path length difference (relative delay) from the first to last strong multipath component becomes smaller, as expected from the geometry. In each figure, $\sigma_{\text {rms }}$ is the RMS-DS of the whole PDP, and $\sigma_{\text {rmsA }}$ is the RMS-DS of part A in each PDP.

The PDPs show that the reflections from the rear door are strong at each position and can be separated from the LOS component. It also can be seen that in tunnel I the duration of reflected multipath from the rear door, as part C in Figure 7 shows, is less than approximately $100 \mathrm{~ns}$, which corresponds to a $30 \mathrm{~m}$ path length difference. Since the tunnel height and width are $3 \mathrm{~m}$ and $5 \mathrm{~m}$, this means that there can be 6 to 10 reflections when the signal propagates from transmitter to receiver from the rear-wall reflections. Thus we note again that 8 reflections were chosen as a reference when we set the ray tracing simulation parameters.

3.2.2. Amplitude Matrix. In general, in small scale MIMO systems, antenna correlation and the MIMO capacity are of primary concern. But in medium or large scale MIMO systems, the received signal strength is also of interest, because when the number of antennas increases, distinct fading across the array may appear in some scenarios. In addition, in confined spaces like indoors and in tunnels, the capacity of any MIMO system is always influenced by specific characteristics of the antenna deployment. Thus novel metrics may be of use to help us understand how the signal propagates; ideally these metrics would be straightforward to compute and intuitive. An amplitude matrix (AM) is introduced here to show the effects of varying the antenna position.

Table 2 lists the number of multipath components using our fixed threshold and the resolved number of multipath 


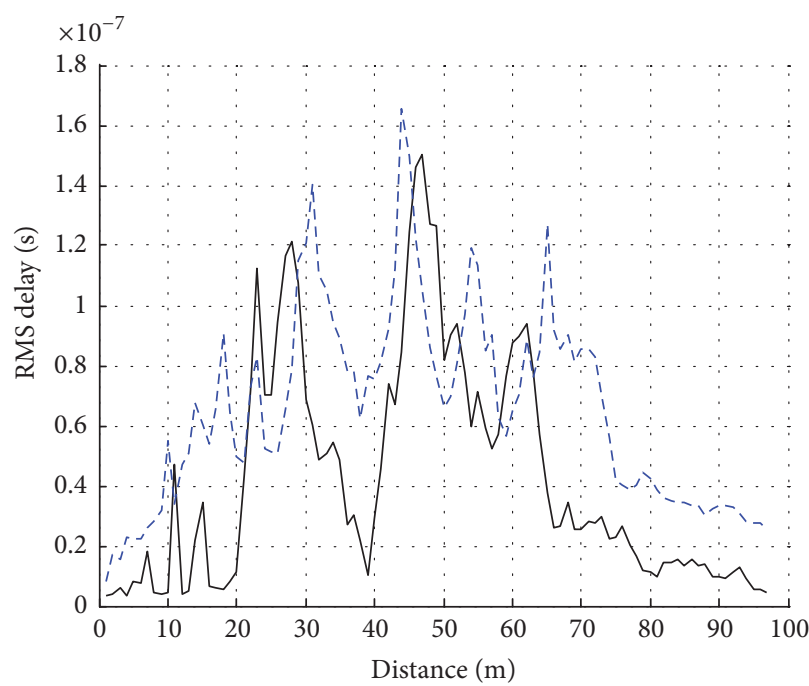

- Measurement

- - - Ray tracing

(a) RMS-DS with rear door reflection

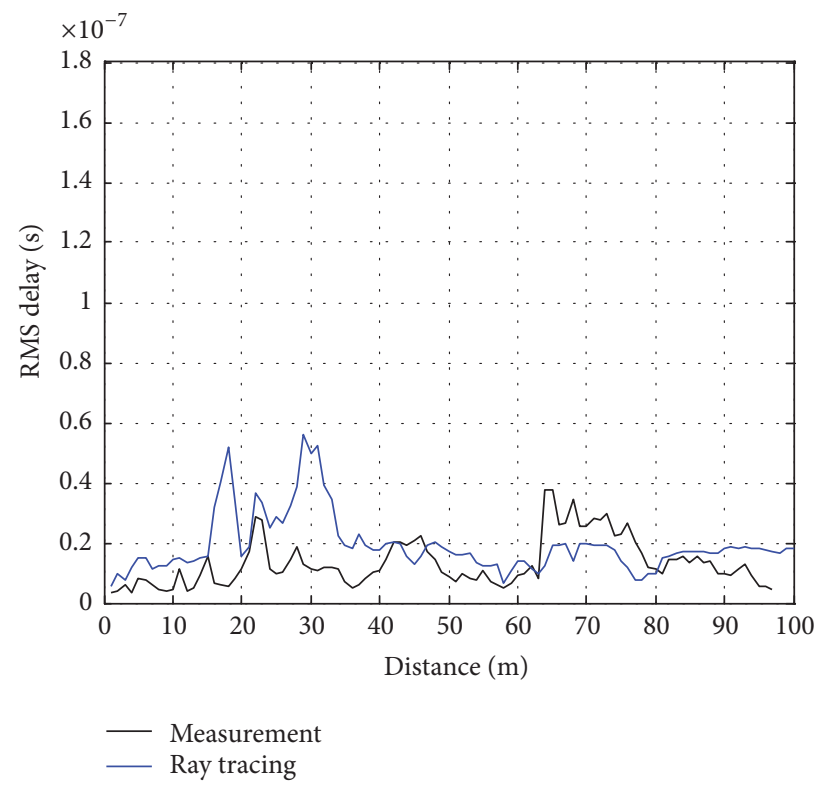

(b) RMS-DS without rear door reflection

FIGURE 5: RMS-DS versus distance for measurement and ray tracing results.

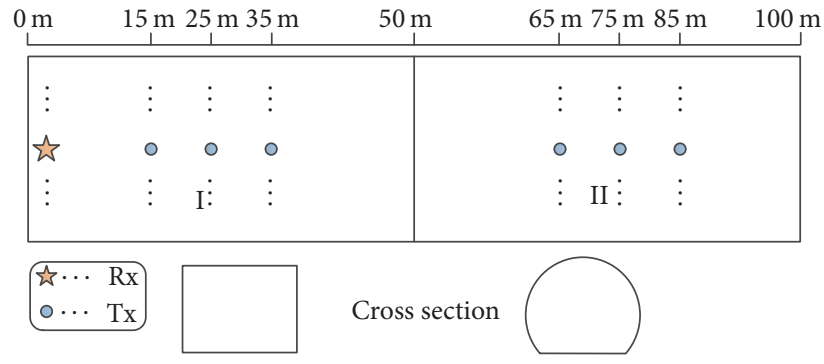

FIGURE 6: Measurement location illustration for virtual MIMO measurements.

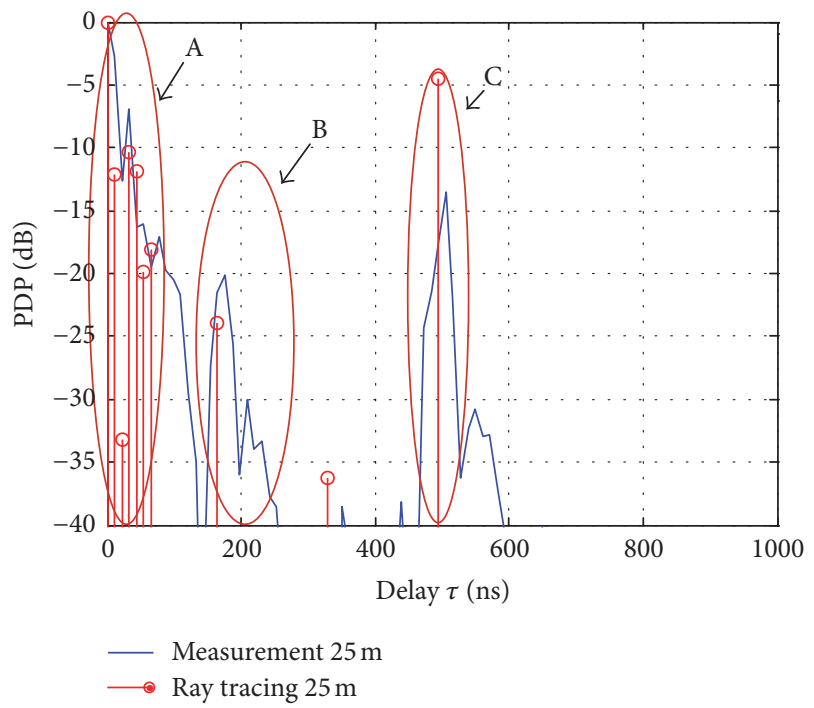

FIGURE 7: A PDP example for the $25 \mathrm{~m}$ link distance.

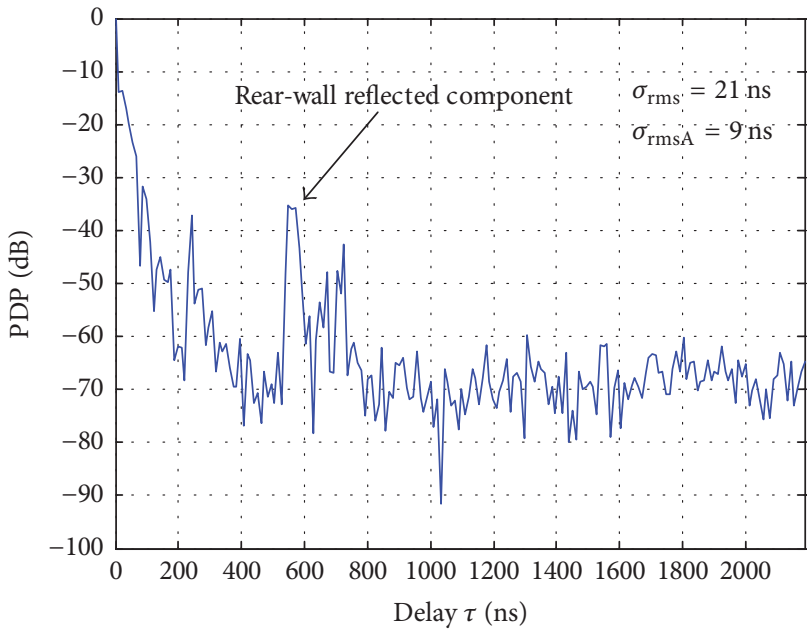

Figure 8: PDP at $15 \mathrm{~m}$.

components extracted by SAGE. The comparison indicates that SAGE algorithm can slightly improve the resolution tunnel scenario. For simplicity multipath components were obtained by fixed threshold method. After removing the noise components, the multipath complex components above the noise floor are combined in the delay domain as

$$
A_{m n}=\left|\sum_{\tau} h_{m n}(\tau)\right|
$$

where $A_{m n}$ is the amplitude of the channel between the $m_{\text {th }}$ Tx antenna and $n_{\text {th }}$ Rx antenna, $m=1, \ldots, M, n=1, \ldots, N$. 


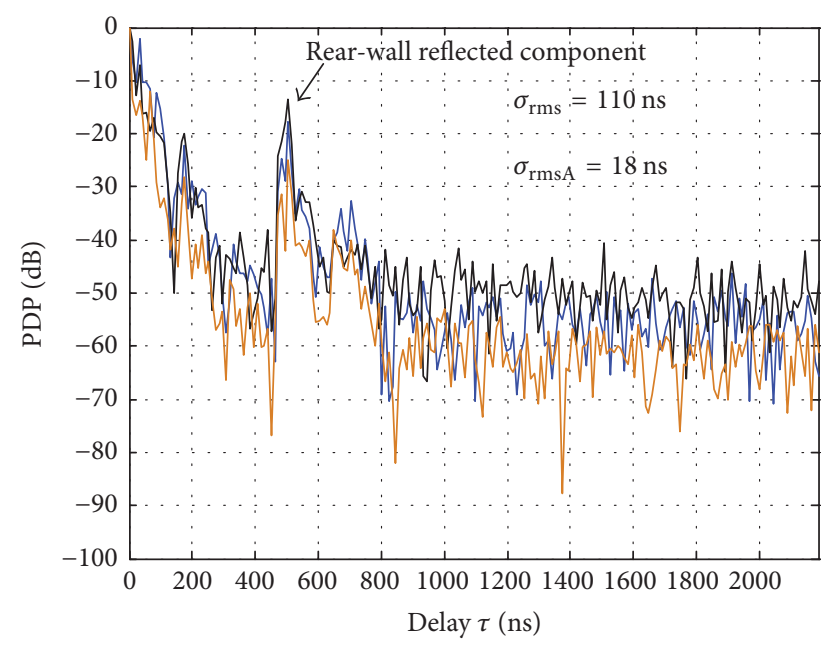

$\begin{aligned}-\mathrm{Tx} & =2.3 \mathrm{~m} \\ -\mathrm{Tx} & =2.4 \mathrm{~m} \\ \mathrm{Tx} & =2.5 \mathrm{~m}\end{aligned}$

FIGURE 9: PDP at $25 \mathrm{~m}$.

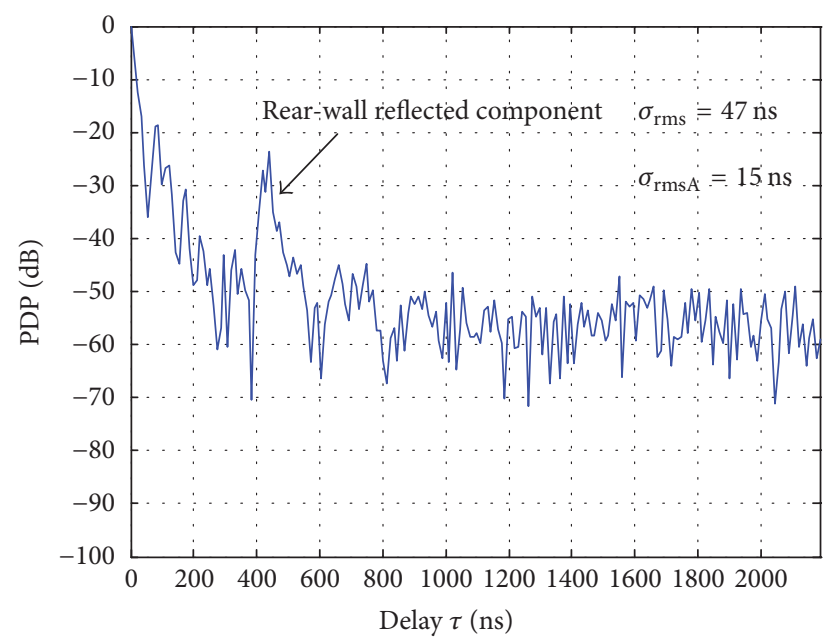

FIGURE 10: PDP at $35 \mathrm{~m}$.

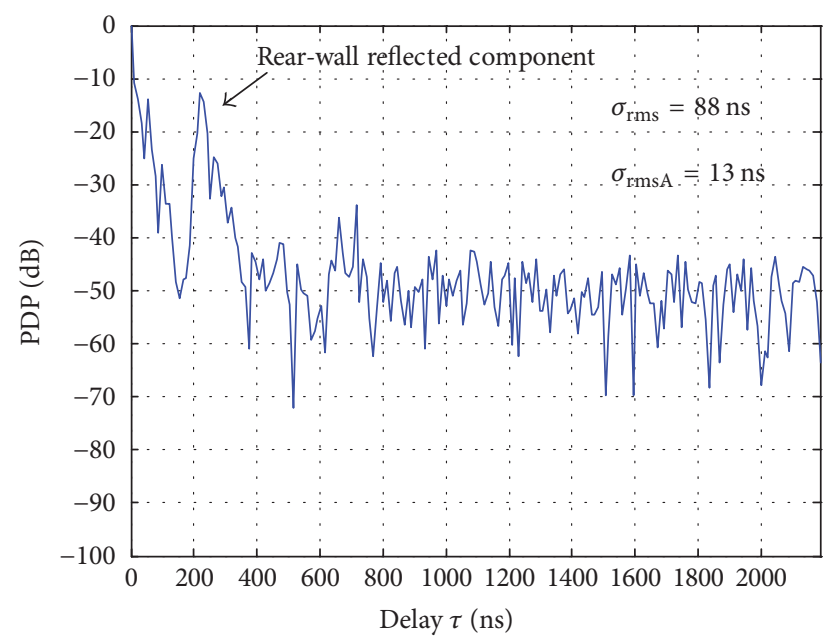

FIgURE 11: PDP at $65 \mathrm{~m}$.

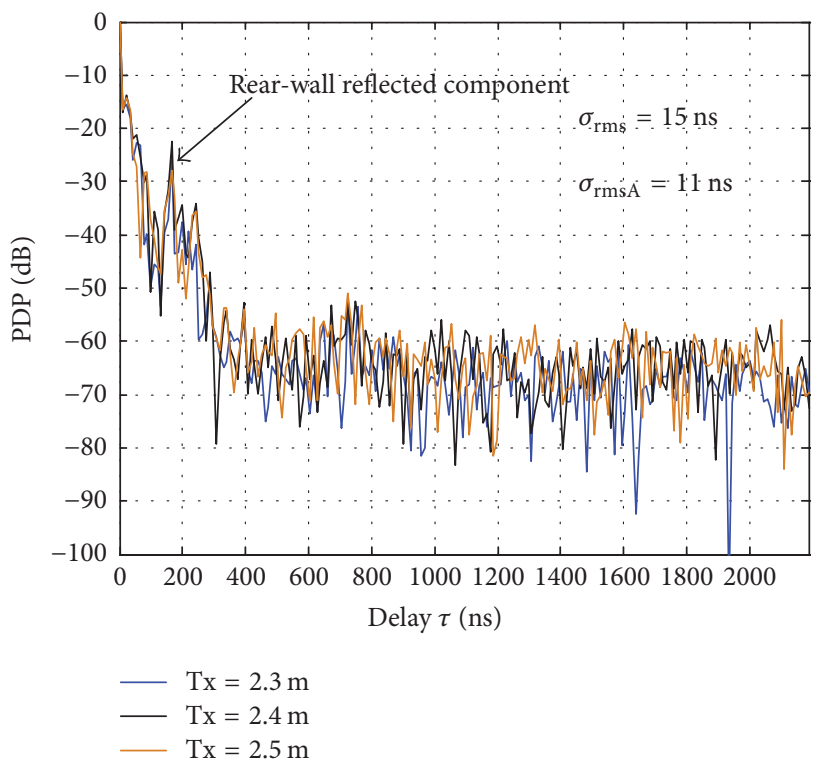

FIGURE 12: PDP at $75 \mathrm{~m}$.

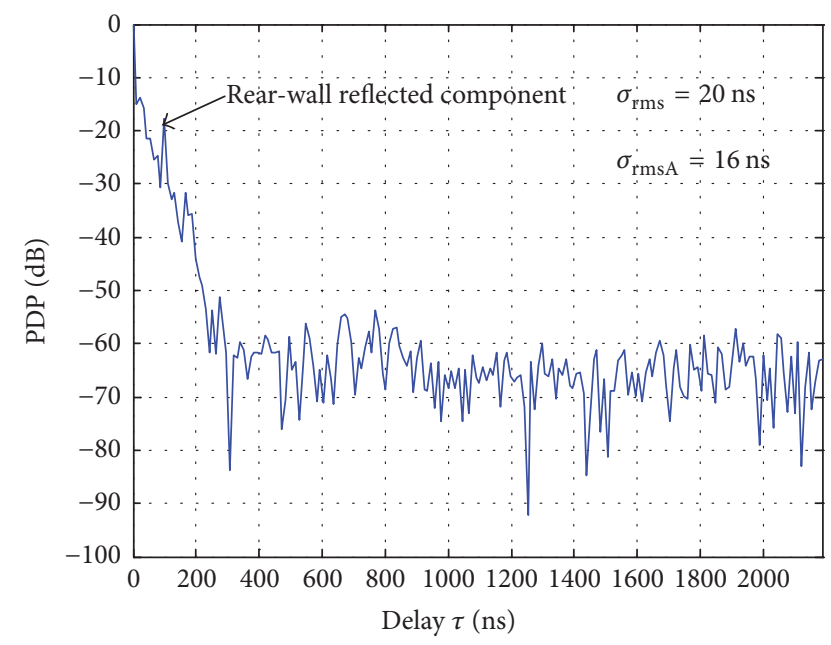

Figure 13: PDP at $85 \mathrm{~m}$.

So the MIMO amplitude matrix $\widetilde{\mathbf{A}}_{M N}$ is given by

$$
\widetilde{\mathbf{A}}_{M N}=\left(\begin{array}{ccc}
A_{11} & \cdots & A_{1 n} \\
\vdots & \ddots & \vdots \\
A_{m 1} & \cdots & A_{m n}
\end{array}\right) .
$$

For purposes of comparison, we use the normalized MIMO amplitude matrix, which is written as

$$
\mathbf{A}_{M N}=\frac{\widetilde{\mathbf{A}}_{M N}}{\max \left(\widetilde{\mathbf{A}}_{M N}\right)} .
$$

The normalized MIMO amplitude matrices are displayed from $15 \mathrm{~m}$ to $85 \mathrm{~m}$ in Figure 14. These show the normalized amplitude matrices of the virtual MIMO array at the various link distances. An interesting phenomenon can be observed: 


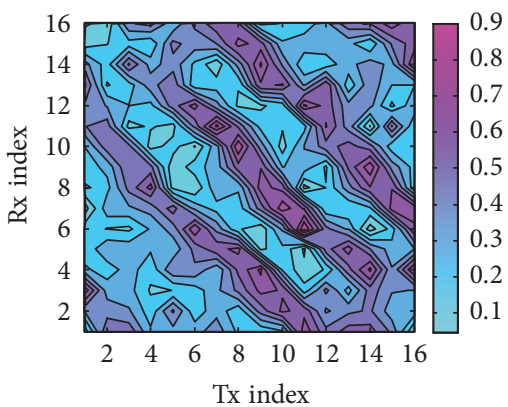

(a)

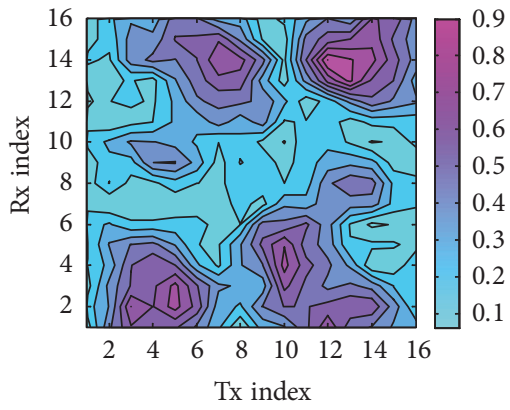

(d)

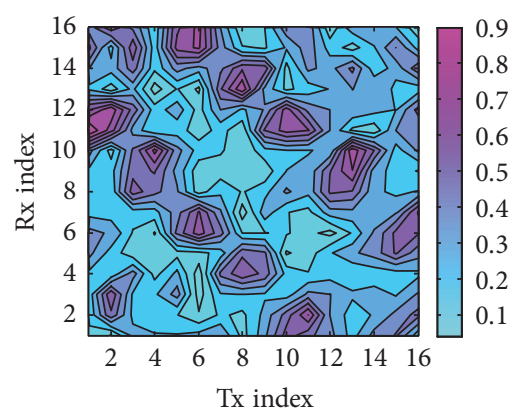

(b)

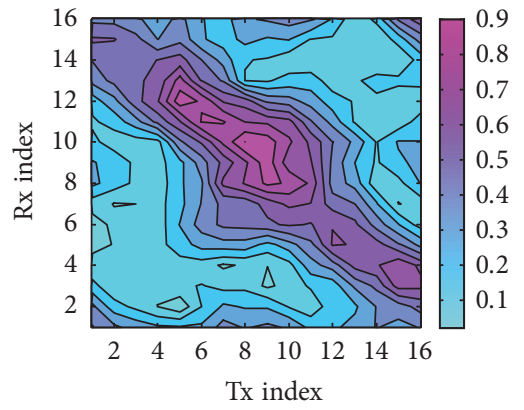

(e)

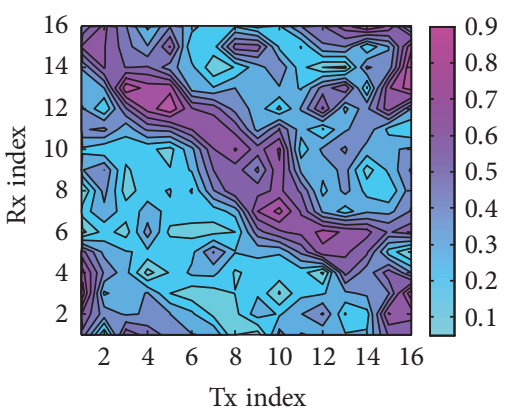

(c)

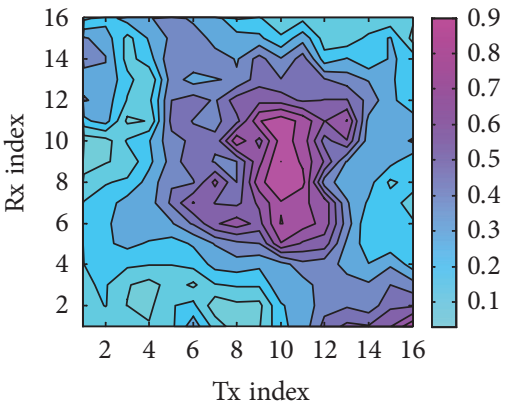

(f)

Figure 14: Measured amplitude matrices at (a) $15 \mathrm{~m}$, (b) $25 \mathrm{~m}$, (c) $35 \mathrm{~m}$, (d) $65 \mathrm{~m}$, (e) $75 \mathrm{~m}$, and (f) $85 \mathrm{~m}$.

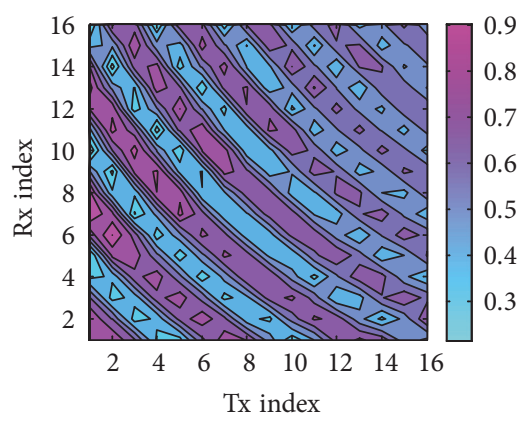

(a)

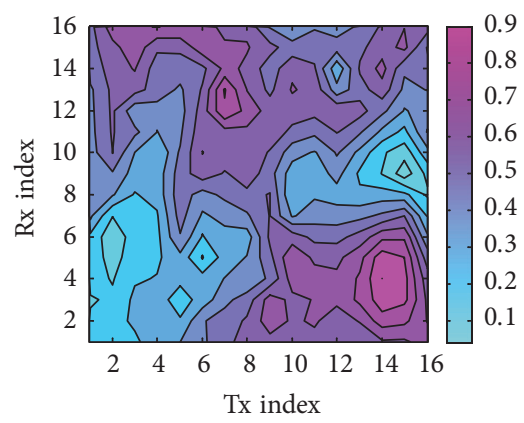

(d)

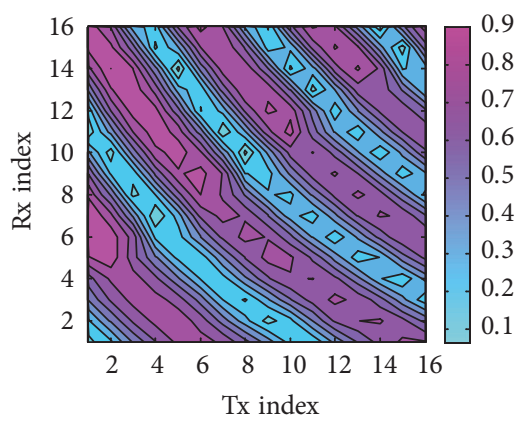

(b)

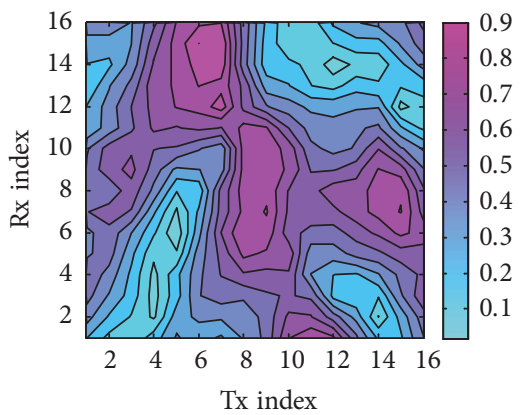

(e)

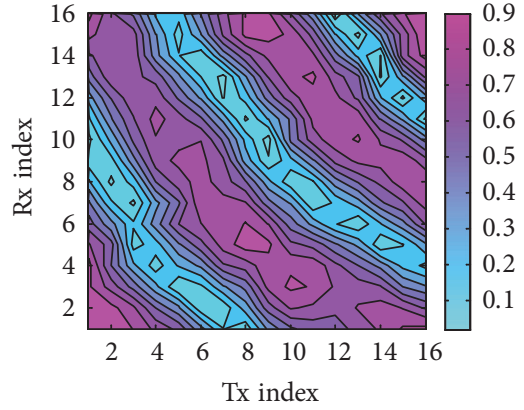

(c)

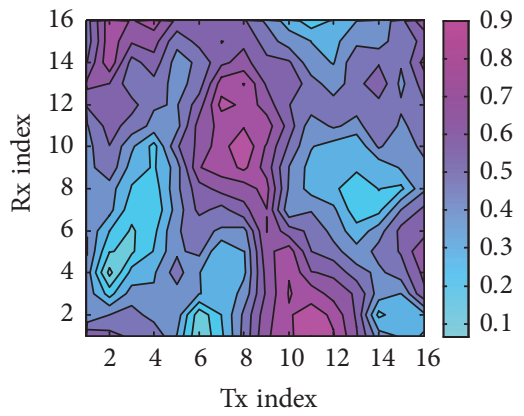

(f)

Figure 15: Simulated (ray tracing) amplitude matrices at (a) $15 \mathrm{~m}$, (b) $25 \mathrm{~m}$, (c) $35 \mathrm{~m}$, (d) $65 \mathrm{~m}$, (e) $75 \mathrm{~m}$, and (f) $85 \mathrm{~m}$.

most of the amplitude matrix plots indicate that the signal is transmitted mainly in a diagonal way, except for the case in Figure 14(d). The corresponding simulation results in Figures 15(a)-15(f) show the same phenomenon even more clearly than in Figures 14(a)-14(f). Figures 16(a) and 16(b) are illustrations of the two types of propagation modes observed from the amplitude matrices at $25 \mathrm{~m}$ and $35 \mathrm{~m}$. It can be concluded, as expected, that reflections of the tunnel walls play a dominant role in the received signal at all link distances; for narrowband signals, also as expected, 


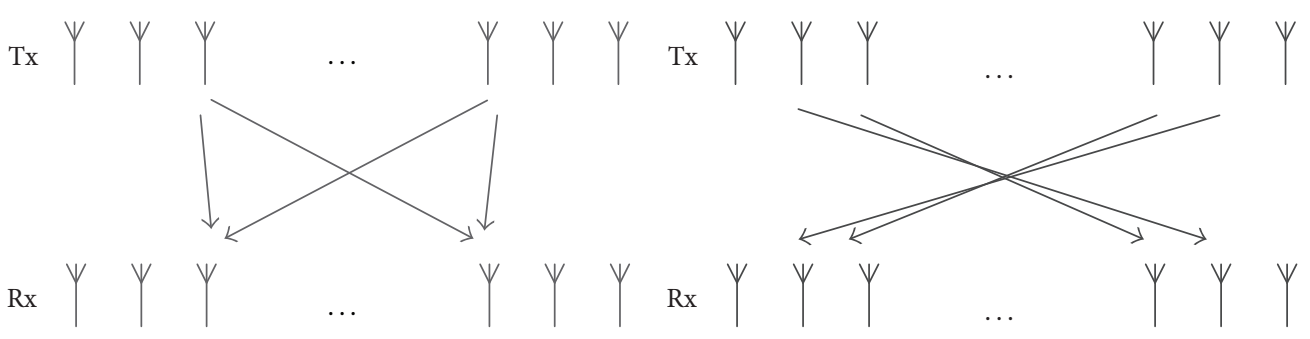

(a) $25 \mathrm{~m}$

(b) $35 \mathrm{~m}$

FIGURE 16: Illustrations of the two propagation modes at $25 \mathrm{~m}$ and $35 \mathrm{~m}$ based upon the amplitude matrices.

the antenna position has a great impact on the received signal strength in these tunnel scenarios. To utilize these propagation characteristics, if the MIMO system is deployed in the tunnel, a suitable antenna selection algorithm may help the communication system reach its maximum capacity.

Ray tracing results for the amplitude matrices are shown in Figure 15. These figures reveal that ray tracing results can only roughly agree with the measurement results. Results in Figure 15 are, by visual inspection, the closest to measurements we have been able to obtain by carefully varying the representation of the tunnel structure and the parameters of the tunnel walls, as previously noted in discussing the PDPs and delay spread. In spite of that, the principal diagonal transmission character at each position is even more strongly present in the simulations. Furthermore, as the link distance increases, the number of diagonal "bands" decreases. We hypothesize that this is because the angular spread decreases with distance.

To help us judge the simulation accuracy, we have computed two metrics to compare the agreement between amplitude matrices of simulations and measurements: the root-mean-square error (RMSE) (11) and vector correlation (12). In (11) and (12) $A$ and $\bar{A}$ are the vectorized versions of the measured amplitude matrix $\mathbf{A}$ and simulated amplitude matrix $\overline{\mathbf{A}}$, where $N_{\mathrm{AM}}$ is total number of elements in the amplitude matrix.

$$
\begin{aligned}
\varepsilon_{\mathrm{RMSE}} & =\sqrt{\frac{\sum_{N}(A-\bar{A})^{2}}{N_{\mathrm{AM}}}}, \\
\varepsilon_{\mathrm{corr}} & =\frac{A \cdot \bar{A}}{\|A\|\|\bar{A}\|} .
\end{aligned}
$$

As an absolute error, it is difficult to judge the simulation accuracy solely from the RMSE values, so the correlation is used here as our primary metric. For these metrics, good agreement between simulation and measurements means that $\varepsilon_{\text {RMSE }}$ is small, and $\varepsilon_{\text {corr }}$ is large (near unity). Table 2 shows the RMSE and correlation results, and the latter are always larger than 0.8 , indicating at least reasonable simulation accuracy.

The results listed in Table 3 show the relative agreement between the measurement and simulation at fixed points in space. Since small scale effects may increase the actual error in such a confined space, an average of the amplitude
TABLE 3: Estimation error metric values versus link distance $(\mathrm{m})$.

\begin{tabular}{ccccccc}
\hline \multicolumn{7}{c}{ Position } \\
& 15 & 25 & 35 & 65 & 75 & 85 \\
\hline Metric & & & & & & \\
$\mathcal{E}_{\text {RMSE }}$ & 0.3626 & 0.3974 & 0.3932 & 0.2933 & 0.3059 & 0.3040 \\
$\boldsymbol{\varepsilon}_{\text {corr }}$ & 0.8377 & 0.8027 & 0.8018 & 0.8533 & 0.8553 & 0.8536 \\
\hline
\end{tabular}

TABLE 4: Estimation error metrics after averaging.

\begin{tabular}{lccc}
\hline Metric & \multicolumn{3}{c}{ Average over } \\
& $\mathrm{Tx}$ & $\mathrm{Rx}$ & $\mathrm{Tx} \& \mathrm{Rx}$ \\
\hline$\varepsilon_{\text {RMSE }}$ & 0.3084 & 0.2792 & 0.2004 \\
$\varepsilon_{\text {corr }}$ & 0.9387 & 0.9333 & 0.9725 \\
\hline
\end{tabular}

over several antennas was computed to see the local area characteristics. Figures 17(a)-17(f) show the measurement and simulated amplitude average results over 4 antennas (1.5 wavelengths) when averaging is done at the $\mathrm{Tx}$ and $\mathrm{Rx}$ and both at Tx and Rx antennas at $25 \mathrm{~m}$. From these figures and the compilation of the estimation error metrics in Table 4 we observe that the local area amplitude matrices match even better than those for the individual array elements, especially after averaging at both $\mathrm{Tx}$ and $\mathrm{Rx}$.

3.2.3. $A O A$ and $A O D$. The azimuth angle characteristics of the channel can be extracted from our linear array measurements [17]. The angle of arrival (AOA) and angle of departure (AOD) are estimated using the space-alternating generalized expectation-maximization (SAGE) algorithm [18], and the results are shown in Table 5. As can be seen, when the Tx and $\mathrm{Rx}$ are both in the rectangular tunnel section (at $15 \mathrm{~m}$, $25 \mathrm{~m}$, and $35 \mathrm{~m}$ ), the range of the AOA and AOD is about 60 degrees. At the longer link distances $(65 \mathrm{~m}, 75 \mathrm{~m}$, and $85 \mathrm{~m}$ ), the range of the AOD decreases and is less than that of the AOA. Interestingly, the range of AOA and AOD both decreases with increasing link distance, as expected, but the AOA is larger than the AOD (except for the shortest link distance of $15 \mathrm{~m}$ ). The RMS angle spread (RMS-AS) is used to quantify the change of the AOA and AOD. It is given by [19]

$$
\sigma_{\theta}=\sqrt{\overline{\theta^{2}}-(\bar{\theta})^{2}}
$$




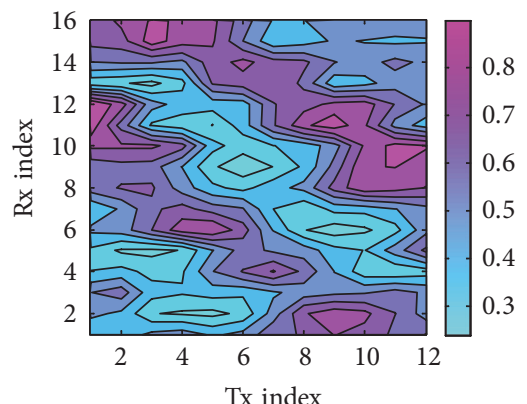

(a) Tx avg

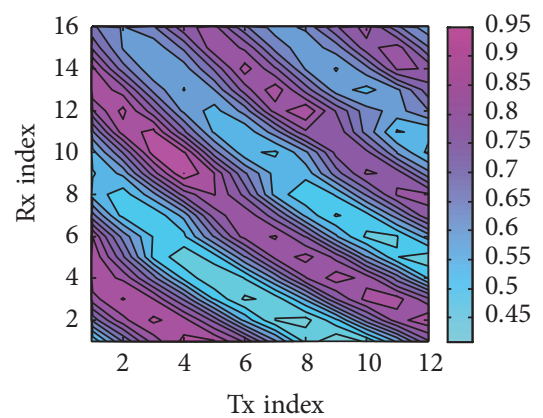

(d) Tx avg

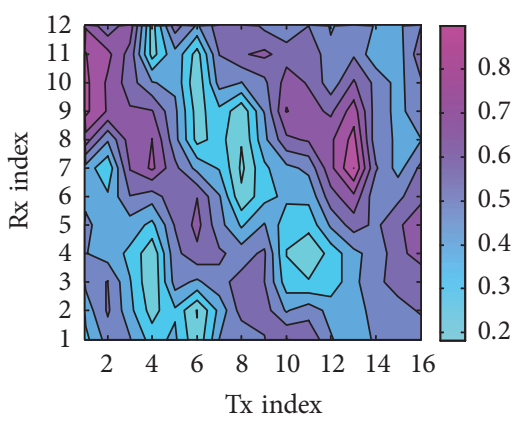

(b) $\mathrm{Rx}$ avg

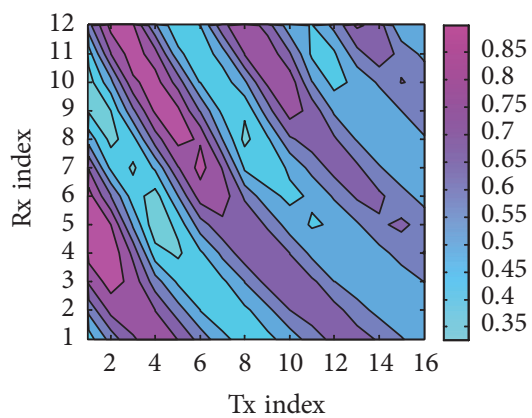

(e) $\mathrm{Rx}$ avg

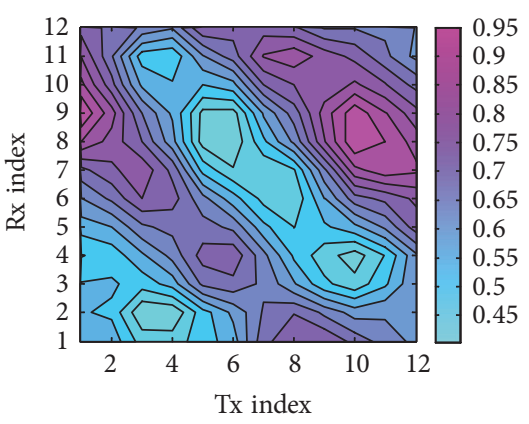

(c) Tx and Rx avg

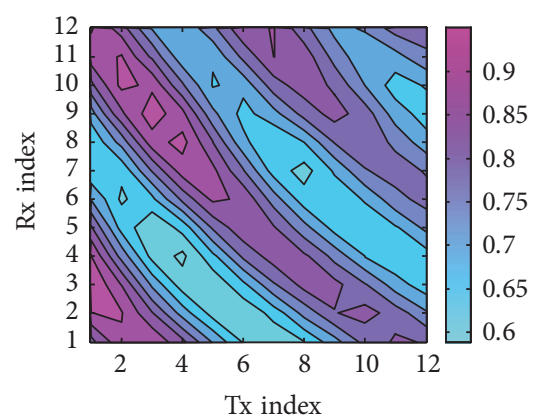

(f) Tx and Rx avg

Figure 17: Measurement (a)-(c) and simulated (d)-(f) amplitude average results over 4 antennas on Tx and $\mathrm{Rx}$ and both on Tx and Rx antennas at $25 \mathrm{~m}$.

where $\overline{\theta^{2}}$ and $\bar{\theta}$ can be written as

$$
\begin{gathered}
\overline{\theta^{2}}=\frac{\sum_{k} P\left(\theta_{k}\right) \theta_{k}^{2}}{\sum_{k} P\left(\theta_{k}\right)}, \\
\bar{\theta}=\frac{\sum_{k} P\left(\theta_{k}\right) \theta_{k}}{\sum_{k} P\left(\theta_{k}\right)},
\end{gathered}
$$

where $\theta_{k}$ and $P\left(\theta_{k}\right)$ are the angle and power of the $k_{\text {th }}$ multipath component. Figure 18 shows the AS versus link distance and summarizes the results of Table 5 . We point out that the received signal is somewhat sensitive to the activities of the testers (i.e., the presence and motion of the humans in the environment): this caused a sudden change of the RMSAS at $75 \mathrm{~m}$, which also can be observed in the prior RMS-DS results and the subsequently provided singular value results. When the Tx and Rx are both located in the rectangular tunnel, the RMS-AS of AOA and AOD is approximately 13 to 16 degrees; when the Tx and $\mathrm{Rx}$ are located in differently shaped sections of the tunnel (and link distance is larger), the RMS-AS of AOA is $\sim 8$ to 10 degrees and the RMS-AS of AOA and $\mathrm{AOD}$ is $\sim 4$ to 7 degrees.

3.2.4. MIMO Capacity. The capacity of a wireless communication link depends on the properties of the complex channel matrix $H$, which is an $M \times N$ dimensional matrix:

$$
H=\left(\begin{array}{ccc}
\widetilde{h}_{11} & \cdots & \widetilde{h}_{1 n} \\
\vdots & \ddots & \vdots \\
\widetilde{h}_{m 1} & \cdots & \widetilde{h}_{m n}
\end{array}\right),
$$

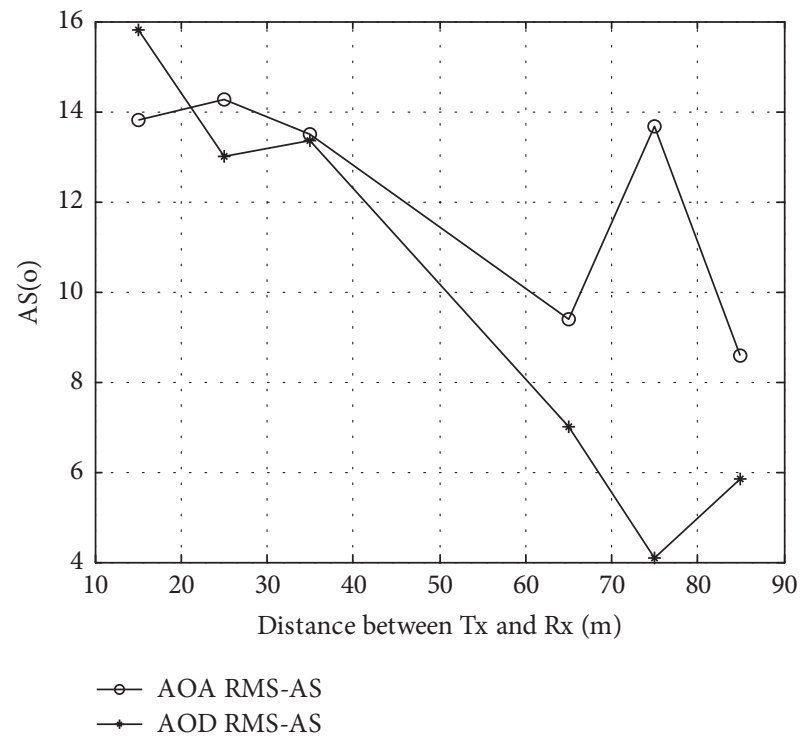

Figure 18: RMS-AS of AOA and AOD versus link distance.

where $\widetilde{h}_{m n}$ is the complex channel coefficient that can be obtained by

$$
\widetilde{h}_{m n}=\sum_{\tau} h_{m n}(\tau) .
$$

In general, a MIMO system's capacity is inversely proportional to the channel correlation between antennas. A low correlation between channels can be achieved by rich 
TABLE 5: AOA and AOD at each position.

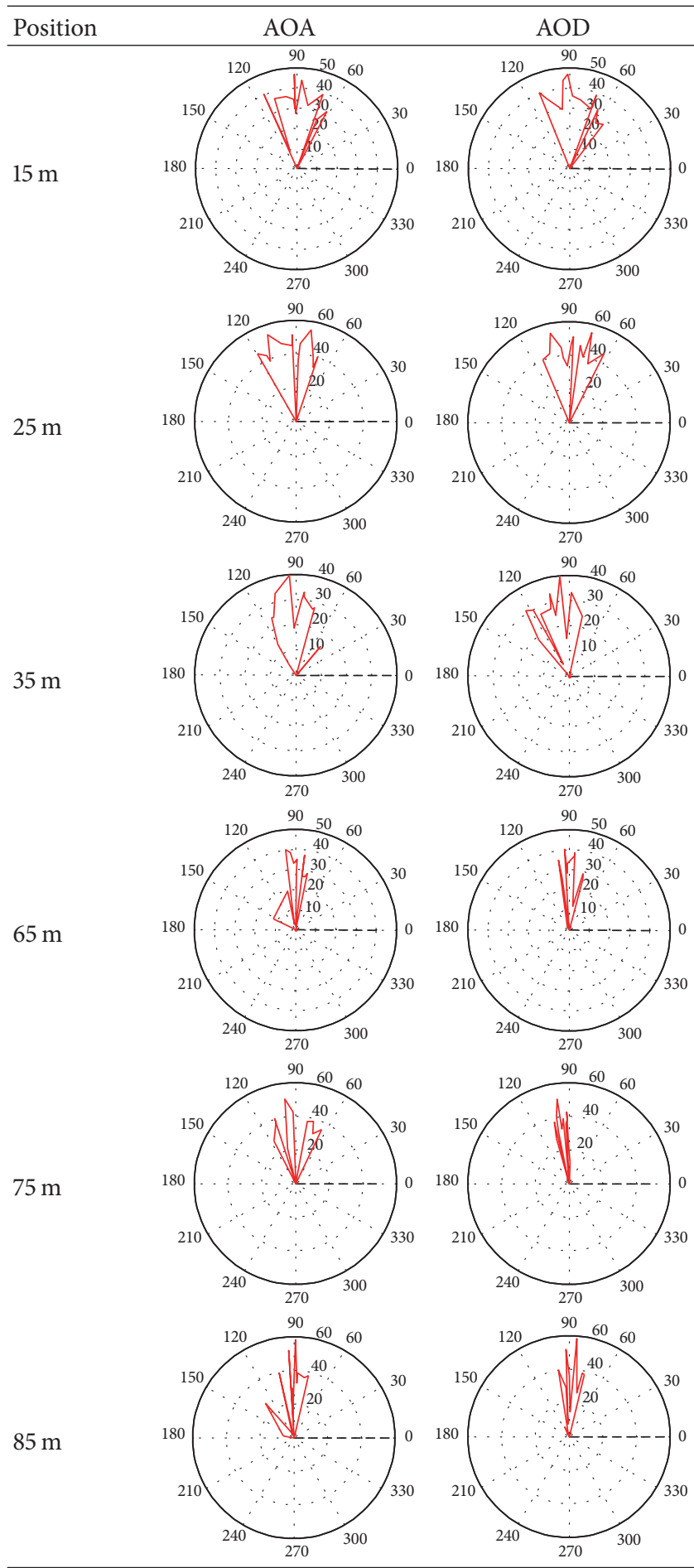

scattering from a large number of reflectors; increasing physical separation between antennas also generally decreases correlation. In tunnel scenarios though, due to the waveguide effect, attenuation of high order modes will reduce the number of the propagating modes, which can be interpreted as being somewhat similar to the key hole effect, and this also reduces the rank of the channel matrix [1] $H$. The singular

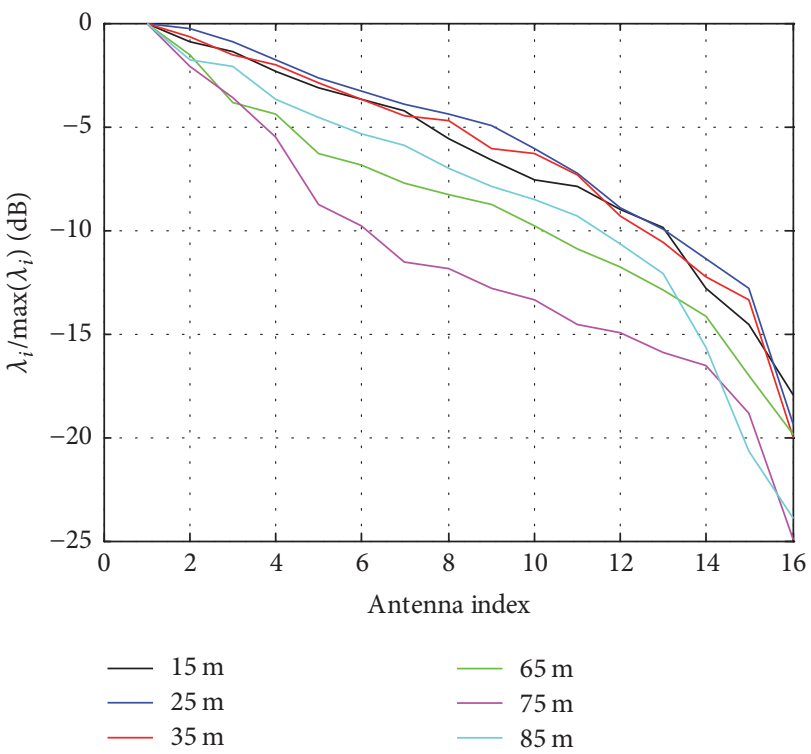

FIGURE 19: Normalized singular values at each value of link distance.

TABLE 6: Ergodic capacity versus link distance.

\begin{tabular}{lcccccc}
\hline $\begin{array}{l}\text { Link } \\
\text { distance (m) }\end{array}$ & 15 & 25 & 35 & 65 & 75 & 85 \\
$\begin{array}{l}\text { Capacity } \\
\text { (bits/s/Hz) }\end{array}$ & 34.2 & 37.29 & 35.85 & 28.65 & 23.1 & 34.63 \\
\hline
\end{tabular}

values of $H$ are often used to quantify the key hole effect, and these will also have direct impact on the MIMO capacity. According to [20], an $M \times N$ matrix $H$ can be put into the form

$$
H=U \Sigma V^{H}
$$

where $U^{M \times M}$ and $V^{N \times N}$ are two distinct unitary matrices and $\Sigma^{M \times M}$ is a positive semidefinite diagonal matrix. The diagonal elements of $\Sigma$ are the singular values of $H$, which are represented by $\lambda_{q}$, where $q=1 \cdots Q$, and $Q$ is equal to the minimum of $M$ and $N$.

Curves in Figure 19 show the normalized singular values, which are obtained by $\lambda_{q} / \max \left(\lambda_{q}\right)$. This figure shows that distance has no obvious or monotonic influence on the normalized singular value, but rather it appears that the crosssectional area has the primary influence: the normalized singular value in the circular tunnel (longer distances) is less than that in the rectangular tunnel (shorter distances).

The maximum capacity of a memoryless $M \times N$ MIMO channel with additive white Gaussian noise can be written as [21]

$$
C=\log _{2}\left[\operatorname{det}\left(I_{M}+\sigma H H^{H}\right)\right],
$$

where $\sigma$ is the average signal-to-noise ratio (SNR), $I_{M}$ is the identity matrix of size $M, H$ is the channel matrix, the superscript $(H)$ denotes Hermitian conjugate, and $\operatorname{det}(\cdot)$ denotes the determinant of a matrix. To illustrate behavior, the ergodic capacity for a SNR of $10 \mathrm{~dB}$ is listed in Table 6, 
TABLE 7: Rayleigh channel from $8 \times 8$ to $16 \times 16$ MIMO capacity.

\begin{tabular}{lccccccccc}
\hline Antenna configuration & $8 \times 8$ & $9 \times 9$ & $10 \times 10$ & $11 \times 11$ & $12 \times 12$ & $13 \times 13$ & $14 \times 14$ & $15 \times 15$ & $16 \times 16$ \\
\hline Capacity (bits/s/Hz) & 21.77 & 24.52 & 27.21 & 29.96 & 32.73 & 35.44 & 38.20 & 40.81 & 43.58 \\
\hline
\end{tabular}

which is the capacity for an outage probability 0.5 . As a comparison, the Rayleigh channel capacities for MIMO array sizes from $8 \times 8$ to $16 \times 16$ are given in Table 7 as well. As with the singular value results, the capacities in the circular tunnel section (longer distances $65-85 \mathrm{~m}$ ) are smaller than those in the rectangular tunnel (shorter distances), with the exception of the $85 \mathrm{~m}$ value. As expected, the capacity results have strong positive correlation with the singular values. And also, by comparing Tables 6 and 7 we can observe that the rank of the channel matrix decreased more in circular tunnel ( 4 to 7 ) than in rectangular tunnel (2 to 3 ). Furthermore, it can be hypothesized that increasing the cross-sectional area appears to improve the MIMO link performance.

\section{Conclusion}

In this article, results of a wide band SISO and virtual MIMO measurement in a tunnel laboratory at a frequency of $1.4725 \mathrm{GHz}$ have been presented. In the SISO case, fairly good agreement was found between the measurement and ray tracing results for path loss and RMS-DS characteristics. The path loss presents strong waveguide effects for distances larger than approximately $45 \mathrm{~m}$. The RMS-DS is near $20 \mathrm{~ns}$ without the rear door reflection and such remote reflectors can obviously increase the RMS-DS in tunnel scenarios.

In the MIMO case, the measurement was conducted at six link distances. The simulated PDPs show fairly good agreement with the measurements; a slight vertical shift of one antenna does not affect the PDP significantly. Amplitude matrices were introduced, and these show strong regularity characteristics at all of the six positions; specifically a strong diagonal transmission characteristic was found. This observation held more strongly when local averaging was applied to the amplitude matrices, and this may be of use in increasing the MIMO system performance. The simulation accuracy of the amplitude matrices based on ray tracing was analyzed as well, and the result also showed acceptable agreement between measurements and simulations. Angular characteristics and channel matrix singular values were also illustrated, and these were in accordance. Finally, although there are rich reflections in the tunnel, the MIMO capacity shows some resemblance to key hole effects according to the narrow angular characteristics and channel matrix singular values.

\section{Conflicts of Interest}

The authors declare that there are no conflicts of interest regarding the publication of this article.

\section{Acknowledgments}

The research was supported in part by the NSFC project under Grant no. 61132003, the Open Research Fund of
National Mobile Communications Research Laboratory, Southeast University, under Grant no. 2014D05, and Beijing Natural Science Foundation project under Grants no. 4152043 and no. 4174102 .

\section{References}

[1] M. Liénard, P. Degauque, J. Baudet, and D. Degardin, "Investigation on MIMO channels in subway tunnels," IEEE Journal on Selected Areas in Communications, vol. 21, no. 3, pp. 332-339, 2003.

[2] M. Liénard, "MIMO channels in tunnels: experimental approach and stochastic model," in in Proceedings of the 10th International Conference on Telecommunications (ICT '03), pp. 1531-1535, Papeete, Tahiti, French Polynesia, French Polynesia, March 2003.

[3] J. A. Valdesueiro, B. Izquierdo, and J. Romeu, "On $2 \times 2 \mathrm{MIMO}$ observable capacity in subway tunnels at C-band: an experimental approach," IEEE Antennas and Wireless Propagation Letters, vol. 9, pp. 1099-1102, 2010.

[4] J.-M. Molina-Garcia-Pardo, J.-V. Rodríguez, and L. Juan-Llacer, "MIMO channel sounder based on two network analyzers," IEEE Transactions on Instrumentation and Measurement, vol. 57, no. 9, pp. 2052-2058, 2008.

[5] E. Masson, Y. Cocheril, M. Berbineau, J.-P. Ghys, V. Hovinen, and A. Roivainen, "MIMO channel characterization in subway tunnel for train-to-wayside applications," in in Proceedings of the 12th International Conference on ITS Telecommunications (ITST '12), pp. 732-736, Taipei, Taiwan, November 2012.

[6] J. Alonso, B. Izquierdo, S. Capdevila, and J. Romeu, "Preliminar propagation and mimo experiments in train tunnels at $5.8 \mathrm{GHz}$," in in Proceedings of the IEEE International Symposium on Antennas and Propagation and USNC/URSI National Radio Science Meeting (APSURSI '09), Charleston, SC, USA, June 2009.

[7] J.-M. Molina-Garcia-Pardo, M. Lienard, A. Nasr, and P. Degauque, "Wideband analysis of large scale and small scale fading in tunnels," in in Proceedings of the 8th International Conference on Intelligent Transport System Telecommunications (ITST '08), pp. 270-273, Phuket, Thailand, October 2008.

[8] L. Zhang, C. Briso, J. R. O. Fernandez et al., "Delay spread and electromagnetic reverberation in subway tunnels and stations," IEEE Antennas and Wireless Propagation Letters, vol. 15, pp. 585$588,2016$.

[9] A. F. Molisch and M. Z. Win, "MIMO systems with antenna selection," IEEE Microwave Magazine, vol. 5, no. 1, pp. 46-56, 2004.

[10] D. G. Dudley, M. Liénard, S. F. Mahmoud, and P. Degauque, "Wireless propagation in tunnels," IEEE Antennas and Propagation Magazine, vol. 49, no. 2, pp. 11-26, 2007.

[11] D. G. Dudley, S. F. Mahmoud, M. Lienard, and P. Degauque, "On wireless communication in tunnels," in in Proceedings of the IEEE Antennas and Propagation Society International Symposium (AP-S '07), pp. 3305-3308, Honolulu, Hawaii, USA, June 2007. 
[12] C. Gentile, F. Valoit, and N. Moayeri, "A raytracing model for wireless propagation in tunnels with varying cross section," in in Proceedings of the IEEE Global Communications Conference (GLOBECOM '12), pp. 5027-5032, Anaheim, Calif, USA, December 2012.

[13] N. Sood, L. Liang, S. V. Hum, and C. D. Sarris, "Ray-tracing based modeling of ultra-wideband pulse propagation in railway tunnels," in in Proceedings of the IEEE International Symposium on Antennas and Propagation and USNC/URSI National Radio Science Meeting (APSURSI '11), pp. 2383-2386, Spokane, Wash, USA, July 2011.

[14] S. Sesia, I. Toufik, and M. Baker, LTE-The UMTS Long Term Evolution: from Theory to Practice, vol. 6, John Wiley and Sons, Hoboken, NJ, USA, 2009.

[15] L. Liu, C. Tao, J. Qiu et al., "Position-based modeling for wireless channel on high-speed railway under a viaduct at $2.35 \mathrm{GHz}$," IEEE Journal on Selected Areas in Communications, vol. 30, no. 4, pp. 834-845, 2012.

[16] T. Zhou, C. Tao, S. Salous, L. Liu, and Z. Tan, "Channel sounding for high-speed railway communication systems," IEEE Communications Magazine, vol. 53, no. 10, pp. 70-77, 2015.

[17] C. Garcia-Pardo, J.-M. Molina-Garcia-Pardo, M. Lienard, D. P. Gaillot, and P. Degauque, "Double directional channel measurements in an arched tunnel and interpretation using ray tracing in a rectangular tunnel," Progress In Electromagnetics Research M, vol. 22, pp. 91-107, 2011.

[18] B. H. Fleury, M. Tschudin, R. Heddergott, D. Dahlhaus, and K. I. Pedersen, "Channel parameter estimation in mobile radio environments using the SAGE algorithm," IEEE Journal on Selected Areas in Communications, vol. 17, no. 3, pp. 434-450, 1999.

[19] N. Czink, E. Bonek, X. Yin, and B. Fleury, "Cluster angular spreads in a MIMO indoor propagation environment," in in Proceedings of the 16th International Symposium on Personal, Indoor and Mobile Radio Communications (PIMRC '05), pp. 664-668, Berlin, Germany, September 2005.

[20] R. A. Horn and C. R. Johnson, Matrix Analysis, Cambridge University Press, New York, NY, USA, 2nd edition, 2012.

[21] G. J. Foschini and M. J. Gans, "On limits of wireless communications in a fading environment when using multiple antennas," Wireless Personal Communications, vol. 6, no. 3, pp. 311-335, 1998. 


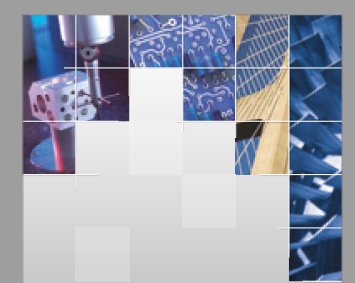

\section{Enfincering}
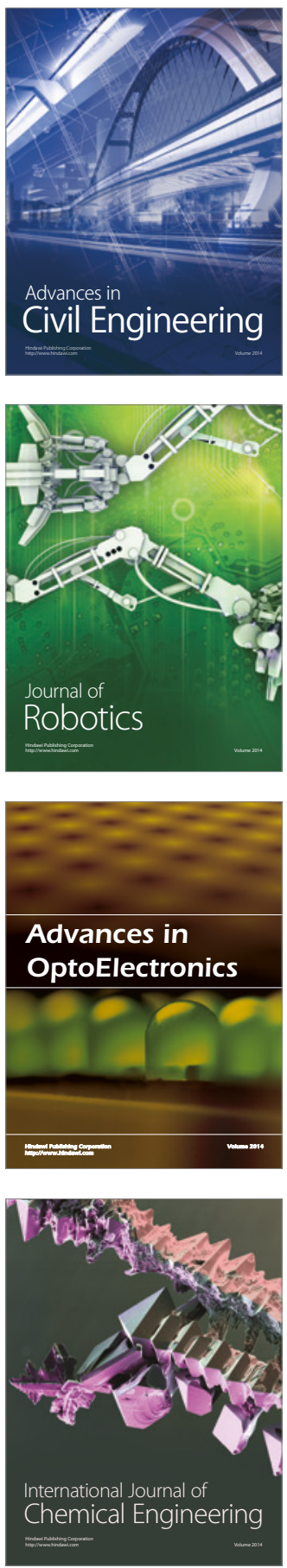

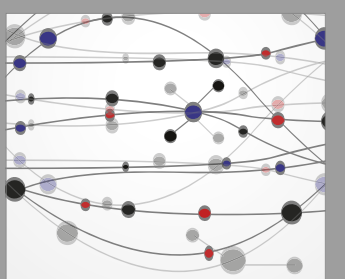

The Scientific World Journal

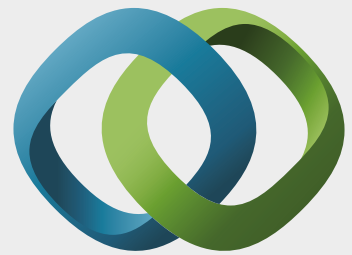

\section{Hindawi}

Submit your manuscripts at

https://www.hindawi.com
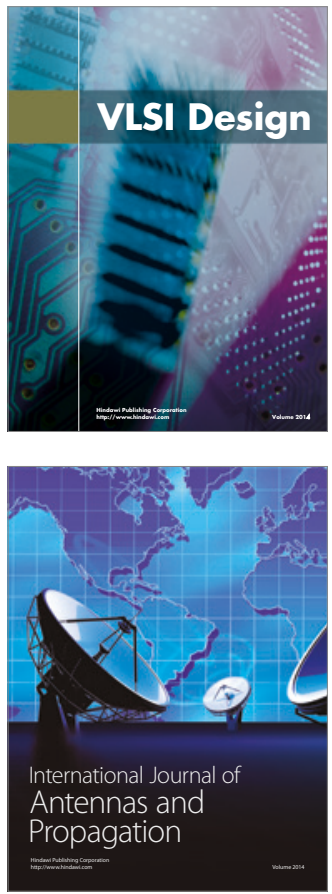

\section{Rotating}

Machinery
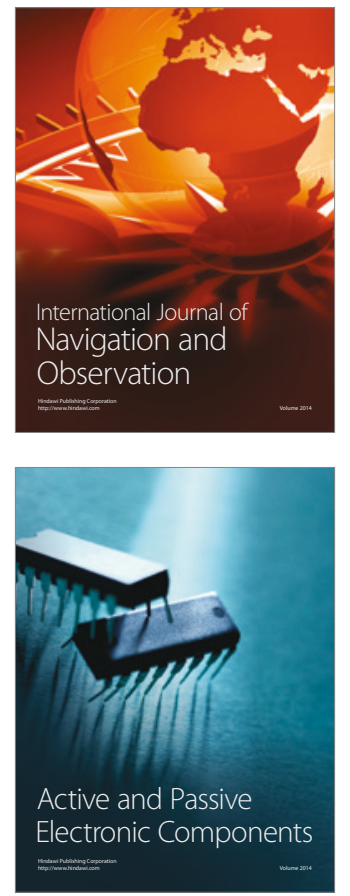
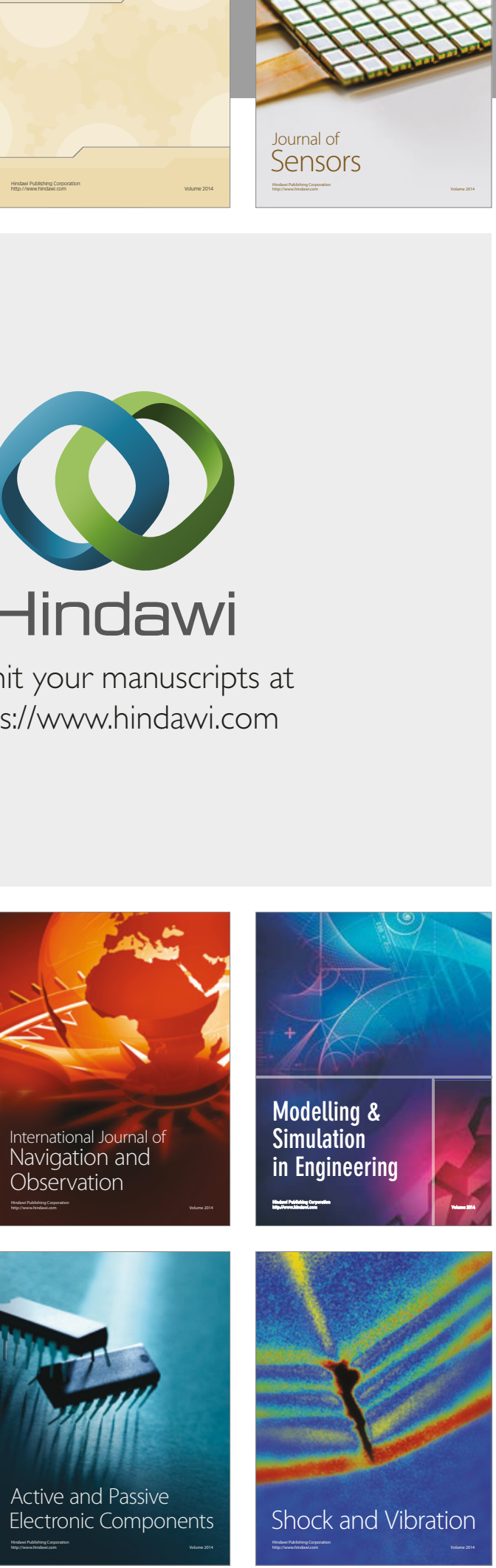
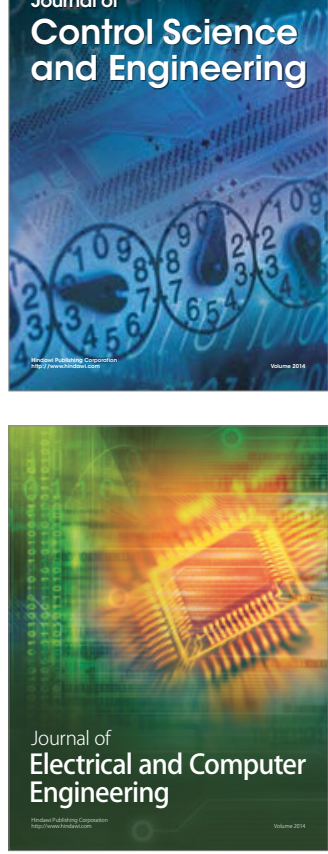

Distributed

Journal of

Control Science

and Engineering
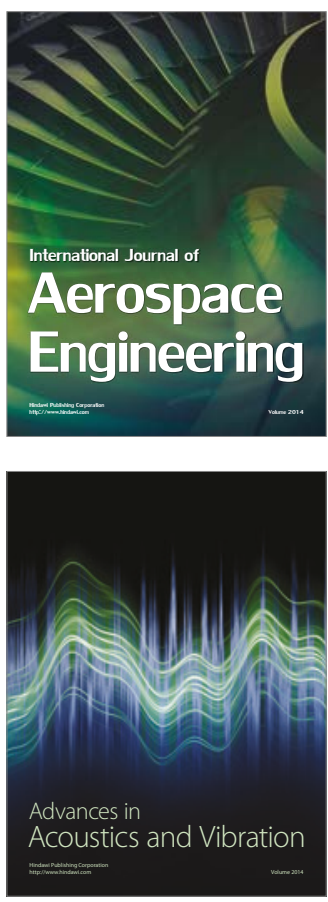

Sensor Networks 